\title{
Treatment of advanced ovarian carcinoma coexistent with peritoneal tuberculosis
}

\author{
AIKO NAGASHIMA $^{1}$, YOSHINARI MATSUMOTO ${ }^{1}$, MASAHIKO OHSAWA $^{2}$ and TOSHIYUKI SUMI $^{1}$ \\ Departments of ${ }^{1}$ Obstetrics and Gynecology, and ${ }^{2}$ Pathology, Graduate School of Medicine, \\ Osaka City University, Osaka 545-8585, Japan
}

Received June 24, 2013; Accepted August 29, 2013

DOI: $10.3892 / \mathrm{mco} .2013 .183$

\begin{abstract}
Primary ovarian carcinoma is often chemosensitive. Therefore, aggressive treatment is recommended for patients with ovarian carcinoma. Peritoneal tuberculosis is rare and may present with symptoms similar to those of advanced ovarian carcinoma. To the best of our knowledge, this is the first report of a case of primary advanced ovarian carcinoma coexistent with peritoneal tuberculosis. The patient had undergone three courses of neoadjuvant chemotherapy with tri-weekly paclitaxel and carboplatin (TC), followed by total abdominal hysterectomy, bilateral salpingo-oophorectomy, omentectomy and pelvic and para-aortic lymphadenectomy. The postoperative pathological examination confirmed the diagnosis of ovarian serous papillary adenocarcinoma coexistent with peritoneal tuberculosis. The patient was started on antituberculous chemotherapy, followed 2 weeks later by four courses of antitumor chemotherapy with weekly TC. The plasma concentration of paclitaxel was measured after the first administration of TC. We considered that rifampicin may enhance the metabolism of paclitaxel, causing the plasma concentration of paclitaxel to decrease. Therefore, rifampicin administration was discontinued on days 1,8 and 15. The patient completed the antitumor and antituberculous chemotherapy and has remained alive and recurrence-free for 5-years. Although rifampicin may enhance the metabolism of paclitaxel, we suggest that it may be possible to administer concurrent antituberculous and antitumor chemotherapy under close observation.
\end{abstract}

\section{Introduction}

Primary ovarian carcinoma is often chemosensitive, particularly the serous papillary adenocarcinoma type. Therefore, patients with advanced ovarian carcinoma often undergo

Correspondence to: Dr Aiko Nagashima, Department of Obstetrics and Gynecology, Graduate School of Medicine, Osaka City University, 1-4-3 Asahimachi, Abeno-ku, Osaka 545-8585, Japan

E-mail:m2068000@med.osaka-cu.ac.jp

Key words: ovarian carcinoma, peritoneal tuberculosis, chemotherapy, paclitaxel, carboplatin aggressive surgical cytoreduction, followed by combination antitumor chemotherapy. Peritoneal tuberculosis is rare and may present with symptoms similar to those of advanced ovarian carcinoma.

The treatment of advanced ovarian carcinoma coexistent with peritoneal tuberculosis requires the administration of chemotherapy effective against both diseases. However, the immunosuppression induced by the antitumor chemotherapy may exacerbate the tuberculosis and the antituberculous chemotherapy may limit the administration of anticancer chemotherapy due to its adverse effects. There are few available case reports of patients treated with antituberculous and antitumor chemotherapy for Müllerian carcinoma (1-3).

The plasma concentration of paclitaxel was measured at various time intervals after the first combined administration of antituberculous and antitumor chemotherapy and further treatment was planned based on these data. To the best of our knowledge, this is the first case report of a patient with primary advanced ovarian carcinoma coexistent with peritoneal tuberculosis.

\section{Materials and methods}

Methods. Staging was performed based on the criteria of the International Federation of Gynecology and Obstetrics (FIGO). After pathological examination had confirmed the diagnosis of ovarian serous papillary adenocarcinoma coexistent with peritoneal tuberculosis, informed consent was obtained from the patient and her family prior to treatment. Blood $(6 \mathrm{ml})$ was collected for analysis at 8 time points. The blood samples were centrifuged (1,600 $\mathrm{x}$ g for $10 \mathrm{~min})$ to separate the plasma, which was stored at $-80^{\circ} \mathrm{C}$ until assayed. The paclitaxel concentrations were measured by a local laboratory (SRL Co., Ltd., Tokyo, Japan), using the high-performance liquid chromatography-ultraviolet method (4).

Case report. A 47-year-old gravida 1, para 1 patient underwent exploratory surgery for ovarian carcinoma by a gynecological surgeon at another hospital, which revealed severe adhesions around a primary large right ovarian tumor and severe carcinomatous peritonitis. A metastatic tumor was identified on the left ovary and left salpingo-oophorectomy was performed. Based on these findings, the patient was diagnosed with serous papillary adenocarcinoma of the ovary, clinical stage IIIc 
and was referred to our hospital. After obtaining informed consent, the patient underwent three courses of neoadjuvant chemotherapy with tri-weekly paclitaxel and carboplatin [TC; paclitaxel $175 \mathrm{mg} / \mathrm{m}^{2}$ body surface area, carboplatin area under the concentration/time curve $(A U C)=6]$. Subsequently, the patient underwent total abdominal hysterectomy, right salpingo-oophorectomy, omentectomy and pelvic and para-aortic lymphadenectomy. There were numerous residual metastases $(<2 \mathrm{~mm})$ on the peritoneum with mild fibrous adhesions around the right adnexa and one 22-mm tumor on the peritoneum over the ileum with mild fibrous adhesions. The microscopic examination of the tumor revealed Langhans-type giant cells, caseous necrosis and lymphocytic infiltration (Fig. 1). The Ziehl-Neelsen staining was negative. The culture and polymerase chain reaction examination of the sputum did not reveal tuberculosis bacteria and the interferon- $\gamma$ release assay (QuantiFERON-TB Gold; Cellestis Ltd, Victoria, Australia) was negative. No evidence of tuberculosis of the lungs or other organs was detected on computed tomography and the diagnosis was confirmed as ovarian serous papillary adenocarcinoma coexistent with peritoneal tuberculosis.

Treatment. The standard antituberculous therapy used in Japan was administered, with isoniazid (300 mg/day), rifampicin (600 mg/day) and ethambutol (1.5 g/day). The patient completed 6 months of ethambutol and 12 months of isoniazid and rifampicin administration, according to the guidelines. After confirming the safety of the antituberculous drugs for 2 weeks, four courses of paclitaxel $\left(70 \mathrm{mg} / \mathrm{m}^{2}\right)$ and carboplatin $(\mathrm{AUC}=2)$ were administered on days 1,8 and 15 every 4 weeks (weekly TC regimen) for ovarian carcinoma. The patient developed an elevation of aspartate aminotransferase and alanine aminotransferase levels and thrombocytopenia. The rifampicin administration was discontinued on days 1,8 , and 15 and the paclitaxel and carboplatin doses were reduced by $20 \%$ from the third course of antitumor chemotherapy onwards.

Follow-up. After the completion of four courses of antitumor chemotherapy, the patient was regularly followed up at our hospital. Imaging examination findings and carbohydrate antigen 125 levels remained within normal limits. The patient has remained alive and recurrence-free for 5 years.

\section{Results}

Plasma concentrations of paclitaxel. The antitumor chemotherapy was initiated after we confirmed the safety of the antituberculous chemotherapy for 2 weeks. In the morning of day 1 of the first course of weekly TC, the patient received antituberculous drugs and $2 \mathrm{~h}$ later paclitaxel was administered over $1 \mathrm{~h}$, followed by carboplatin over $1 \mathrm{~h}$. For pharmacokinetic analysis of paclitaxel, blood samples were collected at 1, 2, 3, $4,9,13,25$ and $49 \mathrm{~h}$ after the initiation of paclitaxel administration. The plasma concentrations of paclitaxel after the first course of antitumor chemotherapy are illustrated in Fig. 2.

\section{Discussion}

It has not been determined whether a regimen of isoniazid and ethambutol affects the pharmacokinetics of paclitaxel.
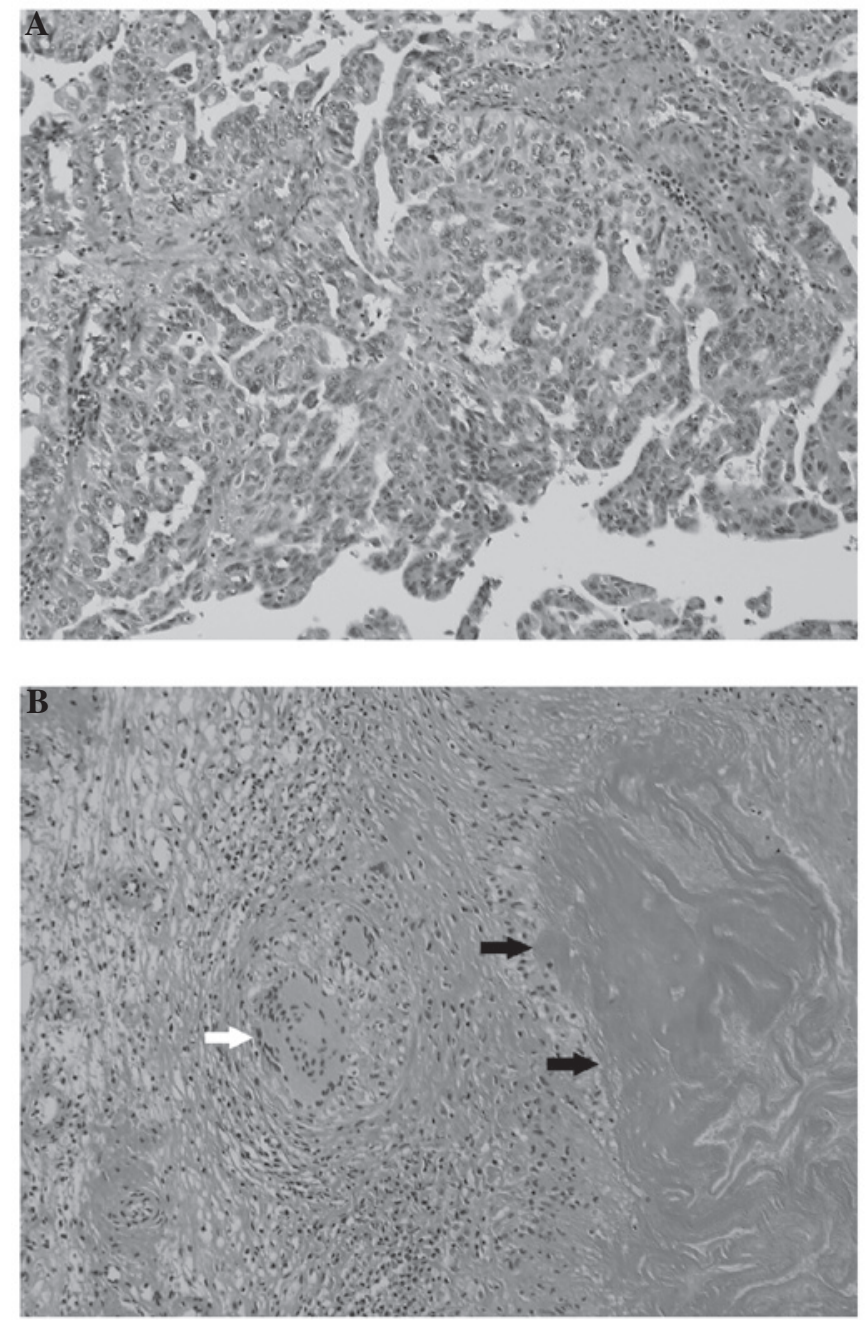

Figure 1.(A) Serous papillary adenocarcinoma of the right ovary. Hematoxylin and eosin staining, original magnification x100. (B) Langhans-type giant cell (white arrow) and caseous necrosis (black arrow) on the peritoneum. Hematoxylin and eosin staining, original magnification x100.

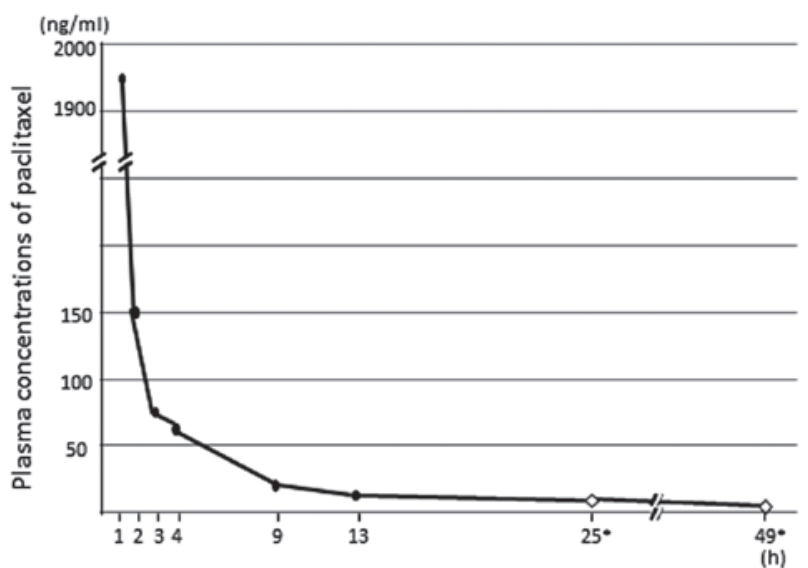

Figure 2. Plasma concentrations of paclitaxel at 1, 2, 3, 4, 9, 13, 25 and $49 \mathrm{~h}$ after the initiation of its administration. "Paclitaxel concentration of 25 and $49 \mathrm{~h}$ were $<10 \mathrm{ng} / \mathrm{ml}$, which is under the measurable value.

The plasma concentration of paclitaxel was measured, since rifampicin induces cytochrome P450 3A4 and may therefore accelerate the metabolism of paclitaxel, resulting in a lower 
concentration and effect of paclitaxel and increasing the risk of hepatic dysfunction.

The plasma concentration of paclitaxel was highest $1 \mathrm{~h}$ after the initiation of its administration and decreased to $<10 \mathrm{ng} / \mathrm{ml}$ after $25 \mathrm{~h}$. A previous study reported that the mean 26-h concentration after a dose of $100 \mathrm{mg} / \mathrm{m}^{2}$ was $0.04 \mu \mathrm{mol} / 1(\sim 34.16 \mathrm{ng} / \mathrm{ml})(5)$ and in the 9 patients for whom 26-h concentration data were available, the plasma concentration was $>0.01 \mu \mathrm{mol} / 1(\sim 8.54 \mathrm{ng} / \mathrm{ml})$, which is the minimum concentration required for its antineoplastic effect (6). We considered that rifampicin may enhance the metabolism of paclitaxel and observed that the concentration of paclitaxel decreased below its effective blood level soon after administration. Therefore, the administration of rifampicin was discontinued on days 1,8 and 15 . However, the plasma concentration of paclitaxel was not measured after the first course of antitumor chemotherapy.

In conclusion, although rifampicin may enhance the metabolism of paclitaxel, we suggest that it may be possible to administer concurrent antituberculous and antitumor chemotherapy under close observation.

\section{References}

1. Chen $\mathrm{CH}$, Huang $\mathrm{CY}$ and Chow SN: Early-stage ovarian carcinoma combined with pulmonary tuberculosis mimicking advanced ovarian cancer: a case report. Int J Gynecol Cancer 14: 1007-1011, 2004

2. Tuon FF, Miyaji KT, de Vidal PM, da Silva LF, Kono A and Franca FO: Simultaneous occurrence of pulmonary tuberculosis and carcinomatous lymphangitis. Rev Soc Bras Med Trop 40: 76-77, 2007.

3. Ingec M, Erdogan F, Kumtepe Y, Isaoglu U, Gundogdu C and Kadanali S: Management of bilateral fallopian tube carcinoma coexistent with tuberculous salpingitis. J Obstet Gynaecol Res 31: 65-67, 2005.

4. Longnecker SM, Donehower RC, Cates AE, et al: High-performance liquid chromatographic assay for taxol in human plasma and urine and pharmacokinetics in a phase I trial. Cancer Treat Rep 71: 53-59, 1987.

5. Seidman AD, Hudis CA, Albanell J, Tong W, Tepler I, Currie V, Moynahan ME, Theodoulou M, Gollub M, Baselga J and Norton L: Dose-dense therapy with weekly 1-hour paclitaxel infusions in the treatment of metastatic breast cancer. J Clin Oncol 16: 3353-3361, 1998 .

6. Jordan MA, Wendell K, Gardiner S, Derry WB, Copp H and Wilson L: Mitotic block induced in HeLa cells by low concentrations of paclitaxel (Taxol) results in abnormal mitotic exit and apoptotic cell death. Cancer Res 56: 816-825, 1996. 